\title{
Avaliação do Uso da Clonidina para Sedoanalgesia de Pacientes sob Ventilação Mecânica Prolongada, Internados em Unidade de Terapia Intensiva*
}

\author{
Evaluate the Clonidine Use for Sedoanalgesia in Intensive \\ Care Unit Patients under Prolonged Mechanical Ventilation
}

Rachel Duarte Moritz', Fernando Osni Machado², Erick Przybysz Pinto², Gil Schmidt Cardoso ${ }^{3}$, Silvia Modesto Nassar ${ }^{4}$

\section{RESUMO}

JUSTIFICATIVA E OBJETIVOS: O controle do desconforto dos pacientes internados em unidade de terapia intensiva (UTI) tem-se tornado essencial na prática da Medicina intensiva. Os fármacos sedoanalgésicos podem influenciar na morbimortalidade do paciente crítico. Agentes $\alpha_{2}$-agonistas podem ter um futuro interessante nas UTI. O objetivo deste estudo foi avaliar a administração da clonidina para a sedoanalgesia de pacientes sob ventilação mecânica (VM) prolongada.

MÉTODO: Estudo de coorte histórico, onde foram selecionados os prontuários dos pacientes internados na UTI entre janeiro e dezembro de 2006, sob sedação, analgesia e ventilação mecânica por período $\geq 7$ dias. Foram anotados os dados demográficos, clínicos e terapêuticos desses pacientes, que foram subdivididos

1. Coordenadora da Residência de Medicina Intensiva do HU-UFSC; Professora Adjunta da UFSC; Mestre em Ciências Médicas da UFSC; Doutora em Engenharia de Produção da UFSC

2. Chefe da UTI do HU-UFSC; Professor Adjunto da UFSC; Mestre em Ciências Médicas da UFSC; Doutor em Medicina - USP

3. Graduando em Medicina da UFSC

4. Professora Titular da UFSC; Mestre em Engenharia de Produção;

Doutora em Engenharia Elétrica

*Recebido da Unidade de Terapia Intensiva do Hospital Universitário Polydoro Ernani de São Thiago da Universidade Federal de Santa Catarina (HU-UFSC), Florianópolis, SC

Apresentado em 05 de dezembro de 2007

Aceito para publicação em 04 de fevereiro de 2008

Endereço para correspondência

Dra. Rachel Duarte Moritz

Rua João Paulo 1929 - Bairro João Paulo

88030-300 Florianópolis, SC

E-mail: rachel@hu.ufsc.br

(C)Associação de Medicina Intensiva Brasileira, 2008 em três grupos: G1 - medicados com clonidina e outros sedoanalgésicos, G2 - medicados com mais de três fármacos sedoanalgésicos, exceto clonidina, e G3 - medicados com midazolam e fentanil. Registrou-se a dose média diária da clonidina, anotando-se, antes da sua administração, 6 e 24 horas após, os valores da freqüência cardíaca e pressão arterial. Para a análise estatística foram utilizados os testes Análise de Variância (ANOVA), $t$ de Student, $\chi^{2}$ sendo considerado significativo quando $p<0,05$.

RESULTADOS: Foram avaliados 55 pacientes. Quinze (27,3\%) pertenciam ao G1, 11 (20\%) ao G2, $29(52,7 \%)$ ao G3. A idade média dos pacientes foi 44 (G1), 50 (G2) e 55 (G3) anos ( $p=N S$ ). O índice APACHE II médio dos grupos foi 18 (G1), 20,4 (G2), 20,7 (G3) ( $p=N S$ ). Os pacientes do $\mathrm{G} 1$ e $\mathrm{G} 2$ permaneceram mais tempo internados na UTI e no hospital $(p<0,05)$. A dose média administrada de clonidina foi $1,21 \pm 0,54 \mu \mathrm{g} / \mathrm{kg} / \mathrm{h}$. Houve diminuição da freqüência cardíaca e da pressão arterial nos pacientes do G1. Esses efeitos não tiveram repercussão clínica nem relação com a dose da clonidina. A mortalidade foi significativamente menor nos pacientes do G1 (20\%) em comparação com os do G2 (54,5\%) e do G3 (62\%).

CONCLUSÕES: A administração da clonidina não acarretou efeitos colaterais importantes nos pacientes estudados. A taxa de mortalidade dos pacientes medicados com clonidina foi significativamente menor.

Unitermos: Analgesia, Clonidina, Sedação, UTI, Ventilação Mecânica.

\section{SUMMARY}

BACKGROUND AND OBJECTIVES: The control of patient discomfort in the intensive care unit (ICU) has become an integral part of critical care practice. 
The sedoanalgesic drugs could influence critically ill patient's morbimortality. Alpha²-adrenoceptor agonists might have an interesting future in ICU. The objective of this study is to evaluate the clonidine use for sedoanalgesia in ICU patients under prolonged mechanical ventilation

METHODS: Historical cohort study. Admitted patient files, January-December 2006, which stayed under mechanical ventilation, analgesia and sedation $\geq 7$ days were analyzed. Demographic, clinical features and therapeutic data concerning analgesia and sedation were remarked. The data allowed classify the patients in three different groups: $\mathrm{G} 1$ = patients that used clonidine and other drugs; G2 = patients that used three or more drugs, without clonidine and G3 = patients that used fentanyl and midazolam. The mean daily doses of infused clonidine were registered, and the values of heat rate $(\mathrm{HR})$, blood arterial pressure (BAP) before starting use of clonidine, after six hours and 24 hours were also registered. Statistical analyzes were performed using Variance Analysis (ANOVA), $t$ tests and $x^{2}$ (significance $\mathrm{p}<0.05$ ).

RESULTS: Were selected 55 patients. Fifteen (27.2\%) belonged to G1, 11 (20\%) belonged to G2 and 29 $(52.7 \%)$ belonged to $\mathrm{G} 3$. The mean age of patients was 44 (G1), 50 (G2) and 56 (G3) ( $p=N S$ ). The mean score APACHE II was 18 (G1), 20.4 (G2) and 20.7 (G3) (p = NS). G1 and G2 patients presented higher ICU lengthof-stay $(p<0.05)$. The mean dose of clonidine used was $1.21 \pm 0.54 \mu \mathrm{g} / \mathrm{kg} / \mathrm{min}$. G1 patients had HR and BAP decreased, however these effects were not considered clinically relevant. The mortality was lower in the patients from G1 (20\%) when compared to G2 (54.5\%) and G3 $(62 \%)(p<0.05)$.

CONCLUSIONS: The clonidine use to analyzed patients did not result in clinical relevant side effects. The lower mortality index in patients that used clonidine was statistical significant.

Key Words: Analgesia, Clonidine, ICU, Mechanical Ventilation, Sedation

\section{INTRODUÇÃO}

As unidades de terapia intensiva (UTI) são ambientes extremamente estressantes, onde a ansiedade é prevalente, a dor freqüente, o repouso difícil e o sono muitas vezes impossível. Sedativos e analgésicos são comumente utilizados em pacientes internados nas UTI, tanto para aumentar a tolerância ao tratamento intensivo quanto para diminuir as suas respostas fisiológicas ao estresse ${ }^{1,2}$.

A abordagem para promover o conforto do paciente é variável. Embora não exista um fármaco ideal para a sedoanalgesia do paciente crítico, a associação de fármacos tem sido amplamente utilizada (co-sedoanalgesia). Esta associação tem como objetivo, além de propiciar o conforto do paciente crítico, diminuir a tolerância e os efeitos colaterais dos fármacos sedoanalgésicos ${ }^{3}$.

Apesar da maioria dos fármacos utilizados para sedoanalgesia serem os mesmos descritos há cerca de três décadas (midazolam, propofol, fentanil, morfina), um novo enfoque tem sido adotado para o manuseio do estresse e para o controle da dor dos pacientes críti$\cos ^{1-8}$. Tem sido também constatado que uma sedoanalgesia adequada influencia na morbimortalidade, no tempo de internação e de ventilação mecânica e no custo do tratamento desses pacientes ${ }^{9,10}$.

Para a sedoanalgesia dos pacientes internados em UTI, os $\alpha_{2}$-agonistas parecem ter um futuro promissor. O efeito hipnótico e antinoceptivo dos $\alpha_{2}$-agonistas são conhecidos desde a década de 1970. São fármacos que agem ativando os receptores $\alpha_{2}$-adrenérgicos, que pertencem à família das proteínas $\mathrm{G}$. O locus coeruleus (LC), onde se localiza a maioria dos receptores, é a principal região do sistema nervoso central envolvido no efeito sedativo dos $\alpha_{2}$-agonistas. Os $\alpha_{2}$-agonistas continuam a promover analgesia e hipnose mesmo com depleção dos neurotransmissores noradrenérgicos. Isto se explica porque estes fármacos também possuem ação positiva em receptores gabaérgicos no LC. Esses receptores possuem atividade noradrenérgica, o que sugere uma íntima relação entre estes dois mecanismos. A analgesia promovida pelos $\alpha_{2}-$ agonistas deve-se à ação desses fármacos ao nível da medula espinhal ${ }^{11-13}$. A clonidina e a dexmedetomidina são os $\alpha_{2}$-agonistas disponíveis na prática clínica. Esses fármacos parecem ter mais efeito sedativo que analgésico ${ }^{14,15}$.

A clonidina, um fármaco utilizado a longa data para o controle da hipertensão arterial, tem sido indicada para o controle de quadros de abstinência e para a otimização do controle da dor e da ansiedade durante procedimentos anestésicos ${ }^{16-23}$. Entretanto, existem poucos estudos que analisam esse fármaco como um sedoanalgésico para o controle da dor e da ansiedade dos pacientes críticos.

O objetivo deste estudo foi avaliar a administração da clonidina para a sedoanalgesia de pacientes sob ventilação mecânica prolongada. 


\section{MÉTODO}

Trata-se de um estudo de coorte histórico, aprovado pelo Comitê de Ética em Pesquisa com Seres Humanos (CEP) da Universidade Federal de Santa Catarina (UFSC), sob o número 011/01.

Foram avaliados os prontuários dos pacientes admitidos na UTI do HU-UFSC no período de janeiro a dezembro de 2006. Foram considerados sedoanalgésicos: midazolam, propofol, morfina, fentanil, clonidina e cetamina. Foram selecionados os dados dos pacientes medicados com esses fármacos por um período igual ou maior que 7 dias. Foram então anotados os dados demográficos, clínicos e terapêuticos desses pacientes, assim como os aspectos relacionados a ação da sedoanalgesia. Foram excluídos os prontuários que se encontravam com dados incompletos, com páginas ou volumes faltando e fatos que impossibilitavam sua coleta fidedigna.

Após a avaliação dos prontuários, os dados foram subdivididos em três grupos. Os pacientes do G1 receberam clonidina associada a outros fármacos sedoanalgésicos; G2 receberam mais de dois sedoanalgésicos, exceto a clonidina. Aos pacientes do G3 foram administrados somente midazolam e fentanil.

Foi registrada a dose média diária administrada de clonidina $(\mu \mathrm{g} / \mathrm{kg} / \mathrm{h})$. Dos pacientes que receberam esse fármaco foram também anotados, antes da administração da clonidina e após 6 e 24 horas, os valores da freqüência cardíaca $(F C)$, da pressão arterial sistólica (PAS) e da diurese. Para a análise estatística foram utilizados os testes ANOVA, $t$ de Student e $\chi^{2}$. Considerouse significativo quando $p<0,05$.

\section{RESULTADOS}

No ano de 2006 foram internados na UTI-HU 350 pacientes. Desses, 221 prontuários apresentavam dados completos. Constatou-se que 173 pacientes foram sedoanalgesiados, isto é, receberam alguma das medicações avaliadas e 48 não foram medicados com os fármacos estudados. Dos pacientes que receberam sedoanalgesia, 55 permaneceram 7 ou mais dias sob ventilação mecânica e portanto, preencheram os critérios de seleção deste estudo. Dos 55 pacientes avaliados, 15 pacientes $(27 \%)$ receberam clonidina associada a outros fármacos sedoanalgésicos, constituindo o G1, ao G2 11 (20\%) e ao G3 29 (52,7\%). As variáveis clínicas e demográficas dos pacientes avaliados estão demonstradas na tabela 1. Nessa tabela pode-se identificar que os pacientes do G3 permaneceram menos tempo internados na UTI e no hospital $(p<0,05)$.

Nas figuras 1 e 2 estão ilustradas as faltas de correlação entre a gravidade do quadro clínico (escore APA$\mathrm{CHE}$ II) e a idade dos pacientes com a mortalidade.

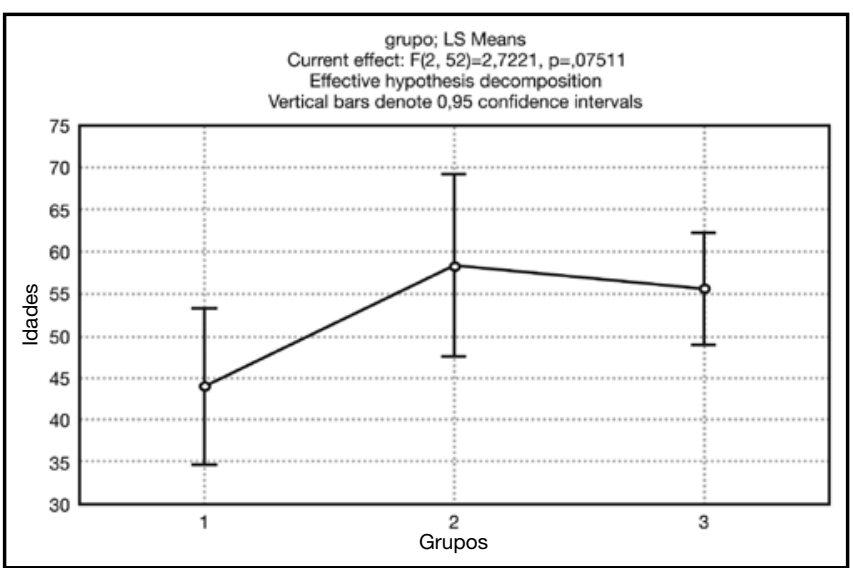

Figura 1 - Correlação entre a Idade e a Mortalidade dos Pacientes

Tabela 1 - Variáveis Clínicas e Demográficas dos Grupos Estudados.

\begin{tabular}{|c|c|c|c|c|}
\hline $\begin{array}{l}\text { Variáveis Clínicas } \\
\text { e } \\
\text { Demográficas }\end{array}$ & $\begin{array}{c}\text { G1 } \\
\text { Co-Sedoanalgesia } \\
+ \\
\text { Clonidina }\end{array}$ & $\begin{array}{c}\text { G2 } \\
\text { Co-Sedoanalgesia }\end{array}$ & $\begin{array}{c}\text { G3 } \\
\text { Fentanil } \\
e \\
\text { Midazolam }\end{array}$ & $\begin{array}{c}\text { Teste } \\
\text { Estatístico }\end{array}$ \\
\hline Idade média (anos) & 44 & 50,07 & 55,68 & NS \\
\hline Desvio-padrão & 19,82 & 20,87 & 15,86 & \\
\hline Mínima & 15 & 15 & 21 & \\
\hline Máxima & 71 & 81 & 83 & \\
\hline \multicolumn{5}{|l|}{ Escore APACHE II } \\
\hline Média & 18 & 20,43 & 20,7 & NS \\
\hline Desvio-padrão & 5,60 & 7,99 & 8,61 & \\
\hline Mínimo & 10 & 10 & 7 & \\
\hline Máximo & 24 & 35 & 31 & \\
\hline Tempo médio de ventilação mecânica (dias) & 11,66 & 11,44 & 10,09 & NS \\
\hline Tempo médio de internação UTI (dias) & 15,73 & 14,33 & 11,65 & $p<0,05$ \\
\hline Tempo médio de internação hospitalar (dias) & 33,66 & 35,19 & 20,89 & $p<0,05$ \\
\hline
\end{tabular}




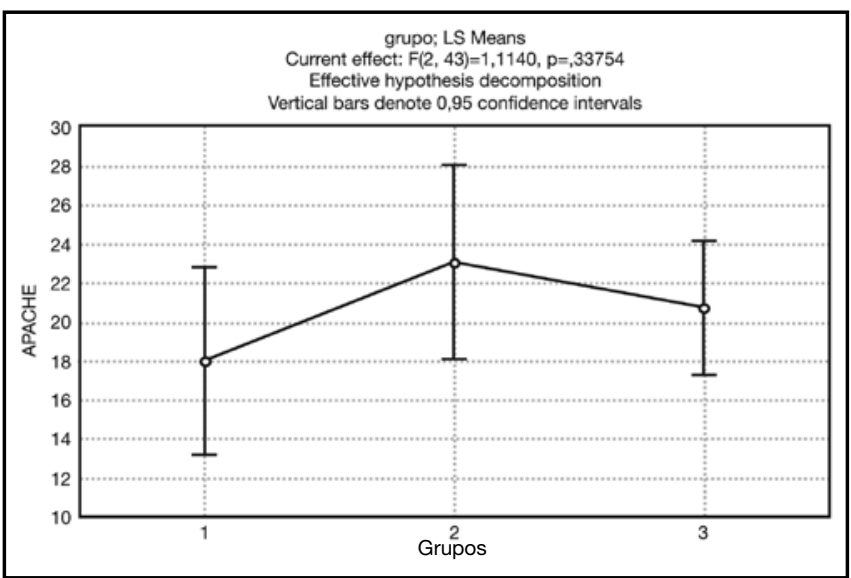

Figura 2 - Correlação entre a Gravidade (Escore APACHE II) e a Mortalidade dos Pacientes

Os pacientes que constituíram o G1 receberam clonidina por tempo médio de $8,13 \pm 7,86$ dias, sendo que o tempo mínimo foi de 2 dias e o máximo de 31 dias. A dose média administrada desse fármaco foi de 1,21 \pm $0,54 \mu \mathrm{g} / \mathrm{kg} / \mathrm{h}$, com variação de 0,64 a $2,35 \mu \mathrm{g} / \mathrm{kg} / \mathrm{h}$.

A comparação da diurese diária dos pacientes antes e após 6 e 24h da administração da clonidina não mostrou diferença significativa do ponto de vista estatístico. Para a avaliação da segurança foram anotadas FC, PAS antes e 6 ou 24h, após a administração da clonidina (Tabela 2). Constatou-se que houve diminuição da FC e da PAS após a sua administração. Somente a medida da FC após $24 \mathrm{~h}$ da introdução de clonidina mostrou diminuição significativa $(p<0,05)$. Entretanto, essa diminuição não foi relacionada à dose da clonidina (Figuras 3 e 4) e não houve re- percussão clínica desses achados. Ressalta-se que a administração da clonidina não foi suspensa em nenhum paciente.

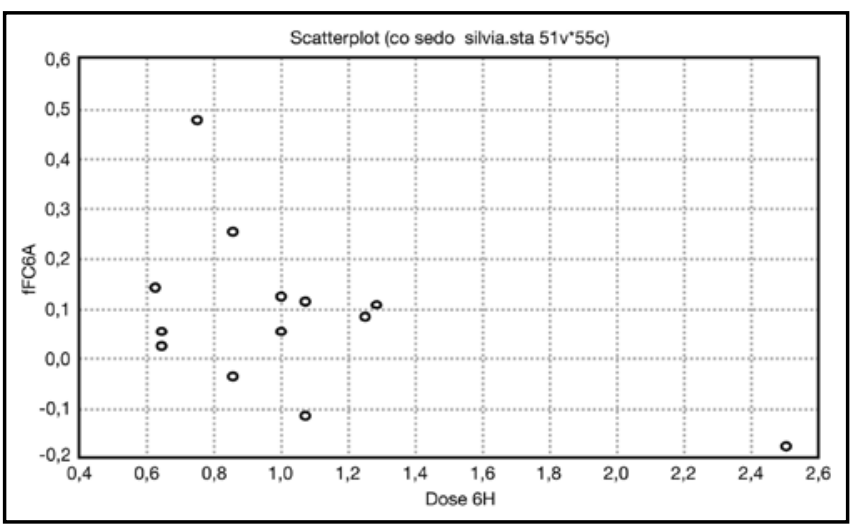

Figura 3 - Correlação entre a Dose da Clonidina e a Freqüência Cardíaca dos Pacientes após 6 Horas.

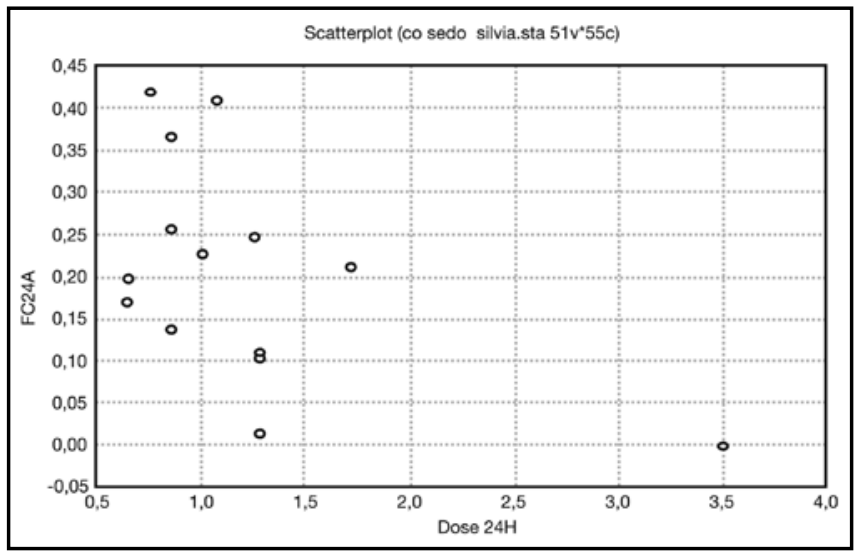

Figura 4 - Correlação entre a Dose da Clonidina e a Freqüência Cardíaca dos Pacientes após 24 Horas.

Tabela 2 - Relação dos Dados Clínicos dos Pacientes Antes e Após 6 ou 24 Horas da Administração da Clonidina.

\begin{tabular}{|c|c|c|c|c|c|c|}
\hline Paciente & $\begin{array}{c}\text { Freqüência Cardíaca } \\
\text { (FC) Antes }\end{array}$ & $\begin{array}{c}\text { FC 6h } \\
\text { após Clonidina }\end{array}$ & $\begin{array}{c}\text { FC 24h } \\
\text { após Clonidina }\end{array}$ & $\begin{array}{c}\text { Pressão Arterial Sis- } \\
\text { tólica (PAS) Antes }\end{array}$ & $\begin{array}{c}\text { PAS 6h } \\
\text { após Clonidina }\end{array}$ & $\begin{array}{c}\text { PAS 24h } \\
\text { após Clonidina }\end{array}$ \\
\hline 1 & 58 & 60 & 52 & 150 & 180 & 170 \\
\hline 2 & 112 & 99 & 66 & 160 & 150 & 160 \\
\hline 3 & 100 & 52 & 58 & 120 & 120 & 150 \\
\hline 4 & 79 & 88 & 50 & 160 & 160 & 120 \\
\hline 5 & 70 & 66 & 69 & 133 & 150 & 170 \\
\hline 6 & 105 & 96 & 79 & 120 & 120 & 120 \\
\hline 7 & 72 & 64 & 64 & 130 & 130 & 110 \\
\hline 8 & 112 & 109 & 93 & 150 & 110 & 130 \\
\hline 9 & 85 & 88 & 67 & 170 & 150 & 160 \\
\hline 10 & 75 & 88 & 75 & 120 & 120 & 70 \\
\hline 11 & 91 & 78 & 73 & 130 & 110 & 90 \\
\hline 12 & 109 & 81 & 81 & 130 & 120 & 110 \\
\hline 13 & 88 & 83 & 68 & 140 & 130 & 120 \\
\hline 14 & 87 & 90 & 75 & 130 & 130 & 110 \\
\hline 15 & 88 & 77 & 68 & 150 & 140 & 140 \\
\hline
\end{tabular}


A taxa de mortalidade dos pacientes estudados está demonstrada na tabela 3. A comparação dos pacientes do G1 com os demais mostrou que a mortalidade foi significativamente menor nesse grupo $(p=0,02)$.

Tabela 3 - Taxa de Mortalidade dos Pacientes nos Grupos Estudados.

\begin{tabular}{lcccc}
\hline Tratamento Sedoanalgésico & \multicolumn{2}{c}{ Óbito } & \multicolumn{2}{c}{ Alta } \\
& $\mathrm{N}$ & $\%$ & $\mathrm{~N}$ & $\%$ \\
\hline G1 = Co-sedoanalgesia e clonidina & 3 & 20 & 12 & 80 \\
G2 = Co-sedoanalgesia & 6 & 54,55 & 5 & 45,45 \\
G3 = Midazolam e fentanil & 18 & 62,06 & 11 & 37,94 \\
\hline
\end{tabular}

\section{DISCUSSÃO}

A sedação e analgesia adequadas são elementos primordiais para o tratamento do paciente crítico submetido à ventilação mecânica ${ }^{1,2,4-8}$. Os fármacos classicamente utilizados para esse fim são os benzodiazepínicos, o propofol e os opióides ${ }^{4-9}$. Nos últimos anos tem havido interesse crescente pela administração de $\alpha_{2}$-agonistas para o controle da dor e ansiedade dos pacientes internados em UTI. A revisão da literatura mostra que tem sido dada ênfase ao estudo da dexmedetomidina para esse fim $^{12-15}$. Poucos trabalhos avaliam a prescrição da clonidina para a co-sedoanalgesia de pacientes críticos ${ }^{1-2}$.

Neste estudo a comparação das variáveis clínicas e demográficas dos pacientes dos diferentes grupos, mostrou que eles eram semelhantes no que concerne a idade e à gravidade do seu quadro clínico. Vários trabalhos revisados por Ostermann e col. ${ }^{5}$ mostraram que quanto mais jovens os pacientes, maior é a dose de sedoanalgésicos exigida ${ }^{9}$. É importante acrescentar que a idade e a presença ou não de comorbidades interferem diretamente no metabolismo dos fármacos ${ }^{4}$. Essas afirmações podem explicar o fato de que os pacientes deste estudo, medicados somente com midazolam e fentanil eram numericamente mais velhos que os demais (55,68 versus 44 e 50,07 anos). Ressalta-se que a comparação estatística mostrou que esses grupos não eram diferentes. Portanto as variáveis idade e gravidade (APACHE II) parecem não ter interferido diretamente na evolução dos pacientes.

Pacientes que necessitam ventilação mecânica invasiva (VMI), precisam de maior quantidade de sedoanalgésicos para o controle da agitação e ansiedade. Tem sido descrito que a sedoanalgesia tem um impacto direto sobre o tempo da VMI dos pacientes críticos, podendo levar a diminuição do seu tempo de extubação sem; entretanto, alterar o seu tempo de internação na
UTI ${ }^{10}$. Neste estudo, apesar de os pacientes que receberam clonidina terem permanecido mais tempo internados, o tempo de VMI foi semelhante nos 3 grupos. Esse fato pode ser importante para a evolução desses pacientes, pois se sabe que existe relação direta entre o tempo de VMI e a incidência de complicações, principalmente as infecciosas ${ }^{24,25}$.

Os efeitos colaterais mais freqüentemente relatados na literatura em relação à administração da clonidina são a hipotensão e a bradicardia ${ }^{17,19}$. Apesar de estes eventos serem geralmente brandos e previsíveis, são responsáveis pela resistência de grande parte dos médicos intensivistas quanto à utilização dos $\alpha_{2}$-agonistas. Após a administração da clonidina, por via sistêmica ou subaracnóidea, a ocorrência de hipotensão foi maior nos pacientes com hipertensão arterial do que nos que apresentam pressão arterial em níveis normais ${ }^{28}$. Um estudo recente mostrou diminuição de PAS em 25\% dos pacientes que foram medicados com clonidina por via peridural, no entanto não houve variações da FC desses pacientes ${ }^{29}$. Neste estudo, somente dois pacientes que receberam clonidina (13\%) apresentaram hipotensão arterial (PAS < 100) após 24 horas da administração desse fármaco. A hipotensão foi controlada com a administração de volume e não acarretou em repercussão clínica importante.

As alterações da FC após a administração dos $\alpha_{2}{ }^{-}$ agonistas, relatadas na literatura, são variáveis ${ }^{30}$. Geralmente a FC é reduzida em diferentes graus após a administração desses fármacos. No entanto, bradiarritmias intensas e persistentes não são freqüentes, mesmo com o uso crônico da clonidina ${ }^{11}$. Neste estudo constatou-se diferença significativa quando foram comparados os níveis de FC antes e após a administração da clonidina. Entretanto, não foi verificada relação entre a dose administrada e a diminuição da FC. Ressalta-se que os níveis de FC não acarretaram em manifestações clínicas. Podem ter contribuído para esse resultado os fatos de que a diminuição da FC tenha também ocorrido pelo melhor controle do estresse do paciente e de que a resposta ao fármaco é individual e não dose-dependente.

Existem evidências que os $\alpha_{2}$-agonistas atuem inibindo a liberação de hormônio antidiurético, antagonizando a ação deste hormônio no túbulo renal e de aumentarem a taxa de filtração glomerular, produzindo um efeito diurético ${ }^{11}$. Neste estudo a comparação da diurese dos pacientes antes e após a administração da clonidina não mostrou diferença. Esse é mais um dado que contribui com o relato de que não houve repercussão clíni- 
ca após a diminuição da PAS e da FC dos pacientes. Uma preocupação dos médicos intensivistas é a de que a administração prolongada dos fármacos sedoanalgésicos possa causar tolerância e provocar mais efeitos colaterais nos pacientes gravemente enfermos. Esse fato também pode influir na morbimortalidade desses pacientes e aumentar o tempo de internação da UTI ou no hospital. Diversas modalidades terapêuticas, têm sido propostas para que sejam evitados esses efeitos. Dentre elas destaca-se a individualização da sedoanalgesia ${ }^{1-3}$, a sua monitoração ${ }^{4,8}$ e a interrupção diária dos fármacos sedoanalgésicos ${ }^{10}$. Neste estudo, constatou-se que os pacientes medicados com clonidina receberam significativamente, doses maiores de outros fármacos sedoanalgésicos (midazolam, fentanil e propofol). Pode-se inferir que esses pacientes eram mais difíceis de sedar. Deve-se relembrar que esse fato não interferiu no tempo de ventilação mecânica que esses pacientes foram submetidos.

Dorman e col. ${ }^{31}$, ao estudarem os efeitos da clonidina sobre a resposta simpática avaliaram 40 pacientes submetidos à cirurgia abdominal de grande porte. Os autores mediram a FC, a PAS e dosaram as catecolaminas plasmáticas, interleucina-6 (IL-6) e o cortisol. Concluíram que a clonidina produz efeitos simpatolíticos, diminuindo a concentração de epinefrina e de norepinefrina em $65 \%$, sem causar alterações nas dosagens de IL-6 e de cortisol. Wallace e col. ${ }^{19}$ estudaram 190 pacientes submetidos à cirurgia com risco de desenvolverem isquemia miocárdica no pós-operatório. Mostraram que a clonidina diminuiu a incidência da mortalidade desses pacientes. Schneemich e col. ${ }^{32}$ apontaram que a clonidina suprime a resposta hiperadrenérgica em pacientes submetidos a endarterectomia de carótica. Von Dossow e col..$^{33}$, por considerarem que os linfócitos $T$ desenvolvem um papel central na resposta imunológica ao trauma, avaliaram 40 pacientes submetidos à cirurgia cardíaca. Mostraram que a clonidina alterou a taxa de subpopulações de linfócitos T no sangue periférico a favor da resposta pró-inflamatória, o que é responsável pela manutenção do balanço imunológico após o ato cirúrgico. Esses estudos suportam os resultados do presente estudo que mostrou significativa diminuição da mortalidade dos pacientes que receberam clonidina.

Os resultados deste estudo permitem afirmar de que a administração da clonidina não acarretou em efeitos colaterais importantes e que houve diminuição significativa da mortalidade dos pacientes medicados com esse fármaco.
O fato dos resultados serem decorrentes de um estudo de coorte retrospectivo pode ser apontado como um fator limitante. Os autores sugerem que novos estudos, prospectivos e aleatórios, sejam realizados para que conclusões definitivas sejam tomadas.

\section{REFERÊNCIAS}

01. Wittbrodt ET, Tietze KJ - Pain control in the intensive care unit. UpToDate, April 2007.

02. Tietze KJ, Wittbrodt ET - Use of sedative medications in critically ill patients. UpToDate, April 2007.

03. Moritz RD - Sedação e analgesia em UTI: velhos fármacos - novas tendências. Rev Bras Ter Intensiva, 2005;17:52-55.

04. Celis-Rodriguez E, Besso J, Birchenall C et al - Clinical practice guideline base don the evidence for the management of sedoanalgesia in the critically ill adult patient. Med Intensiva, 2007;31:428-471.

05. Ostermann ME, Keenan SP, Seiferling RA et al - Sedation in the intensive care unit: a systematic review. JAMA. 2000;283:1451-1459.

06. De Jonghe B, Bastuji-Garin S, Fangio P et al - Sedation algorithm in critically ill patients without acute brain injury Crit Care Med, 2005;33:120-127.

07. Vender JS, Szokol JW, Murphy GS et al - Sedation, analgesia, and neuromuscular blockade in sepsis: an evidence-based review. Crit Care Med, 2004;32:(Suppl11):S554-S561.

08. Jacobi J, Fraser GL, Coursin DB et al - Clinical practice guidelines for the sustained use of sedatives and analgesics in the critically ill adult. Crit Care Med, 2002;30:119-141.

09. Kress JP, Hall JB - Sedation in the mechanically ventilated patient. Crit Care Med, 2006;34:2541-2546.

10. Kress JP, Pohlman AS, O'Connor MF et al - Daily interruption of sedative infusions in critically ill patients undergoing mechanical ventilation. $\mathrm{N}$ Engl J Med, 2000;342:1471-1477.

11. Moritz RD - Alfa 2-agonistas: uma nova opção para a sedação e analgesia em UTI? Rev Bras Ter Intensiva, 2000;12:1773-1781.

12. Pandharipande P, Ely EW, Maze M - Dexmedetomidine for sedation and perioperative management of critically ill patients. Semin Anesth, 2006;25:43-50.

13. Szumita PM, Baroletti SA, Anger KE et al - Sedation and analgesia in the intensive care unit: evaluating the role of dexmedetomidine. Am J Health Syst Pharm, 2007;64:37-44.

14. MacLaren R, Forrest LK, Kiser TH - Adjunctive dexmedetomidine therapy in the intensive care unit: a retrospective assessment of impact on sedative and analgesic requirements, levels of sedation and analgesia, and ventilatory and hemodynamic parameters. Pharmacotherapy. 2007;27:351-359.

15. Venn M, Newman J, Grounds M - A phase II study to evaluate the efficacy of dexmedetomidine for sedation in the medical intensive care unit. Intensive Care Med, 2003;29:201-207.

16. Stanley KM, Worrall CL, Lunsford SL et al - Experience with an adult alcohol withdrawal syndrome practice guideline in internal medicine patients. Pharmacotherapy. 2005;25:1073-1083.

17. Schneemilch CE, Bachmann H, Ulrich A et al - Clonidine decreases stress response in patients undergoing carotid endarterectomy under regional anesthesia: a prospective, randomized, double-blinded, placebo-controlled study. Anesth Analg, 2006;103:297-302.

18. Fernandez-Galinski S, Bermejo S, Mansilla R et al - Comparative assessment of the effects of alfentanil, esmolol or clonidine when used as adjuvants during induction of general anaesthesia. Eur J Anaesthesiol, 2004;21:476-482.

19. Wallace AW, Galindez D, Salahieh A et al - Effect of clonidine on cardiovascular morbidity and mortality after noncardiac surgery. Anesthesiology, 2004;101:284-293 (repetida na 32).

20. Watanabe T, Inagaki $Y$, Ishibe $Y$ - Clonidine premedication effects on inhaled induction with sevoflurane in adults: a prospective, double-blind, randomized study. Acta Anaesthesiol Scand, 2006;50:180-187.

21. Peduto VA, Pisanu GM, Piga M - Midazolam, propofol and clonidine for sedation and control of autonomic dysfunction in severe generalized tetanus. Minerva Anestesiol, 1993;59:171-178. 
22. Spies CD, Dubisz N, Neumann T et al - Therapy of alcohol withdrawal syndrome in intensive care unit patients following trauma: results of a prospective, randomized trial. Crit Care Med, 1996;24:414-422.

23. Bohrer $\mathrm{H}$, Bach $\mathrm{A}$, Layer $\mathrm{M}$ et al - Clonidine as a sedative adjunct in intensive care. Intensive are Med, 1990;16:265:266.

24. Dodek P, Keenan S, Cook D et al - Evidence-based clinical practice guideline for the prevention of ventilator-associated pneumonia. Ann Intern Med, 2004;141:305-313.

25. Rello J, Ollendorf DA, Oster G et al - Epidemiology and outcomes of ventilator-associated pneumonia in a large US database. Chest, 2002;122:2115-2121.

26. Dobrydnjov I, Axelsson K, Gupta A et al - Improved analgesia with clonidine when added to local anesthetic during combined spinal-epidural anesthesia for hip arthroplasty: a double-blind, randomized and placebo-controlled study. Acta Anaesthesiol Scand, 2005;49:538-545.

27. Altan A, Turgut N, Yildiz F et al - Effects of magnesium sulphate and clonidine on propofol consumption, haemodynamics and postoperative recovery. Br J Anaesth, 2005;94:438-441.

28. Fonseca NM, Oliveira CA - Effects of combined clonidine and $0.5 \%$ hyperbaric bupivacaine on spinal anesthesia. Rev Bras Anestesiol, 2001;51:483-492.

29. Schnaider TB, Vieira AM, Brandão ACA et al - Efeito analgésico intraoperatório da cetamina, clonidina ou dexmedetomidina, administradas por via peridural, em cirurgia de abdômen superior. Rev Bras Anestesiol, 2005;55:525-531.

30. Riker RR, Fraser GL - Adverse events associated with sedatives, analgesics, and other drugs that provide patient comfort in the intensive care unit. Pharmacotherapy, 2005;25:8S-18S.

31. Dorman T, Clarkson K, Rosenfeld BA et al - Effects of clonidine on prolonged postoperative sympathetic response. Crit Care Med, 1997;25:1147-1152.

32. Schneemilch CE, Bachmann H, Ulrich A et al - Clonidine decreases stress response in patients undergoing carotid endarterectomy under regional anesthesia: a prospective, randomized, double-blinded, placebo-controlled study. Anesth Analg, 2006;103:297-302.

33. Von Dossow V, Baehr N, Moshirzadeh M et al - Clonidine attenuated early proinflammatory response in T-cell subsets after cardiac surgery. Anesth Analg, 2006;103:809-814. 